\title{
THE LIMITING FAILURE RATE FOR A CONVOLUTION OF LIFE DISTRIBUTIONS
}

\author{
HENRY W. BLOCK, ${ }^{* * *}$ University of Pittsburgh \\ NAFTALI A. LANGBERG, ${ }^{* * *}$ Haifa University \\ THOMAS H. SAVITS, ${ }^{*}$ University of Pittsburgh
}

\begin{abstract}
In this paper we investigate the limiting behavior of the failure rate for the convolution of two or more life distributions. In a previous paper on mixtures, Block, Mi and Savits (1993) showed that the failure rate behaves like the limiting behavior of the strongest component. We show a similar result here for convolutions. We also show by example that unlike a mixture population, the ultimate direction of monotonicity does not necessarily follow that of the strongest component.
\end{abstract}

Keywords: Reliability; failure rate function; increasing failure rate; decreasing failure rate; convolution

2010 Mathematics Subject Classification: Primary 62N05

Secondary $60 \mathrm{~K} 10$

\section{Introduction}

Two of the most important operations in reliability theory are mixtures and convolutions of distributions. For extensive discussions concerning mixtures of distributions and the shape of their failure rate functions, see the monographs of Lai and Xie (2006) and Marshall and Olkin (2007). In this paper, based on the corresponding results for mixtures, we discuss similar results for convolutions.

Convolutions correspond to instantaneous replacement of failed components in mechanical or electrical systems. Mixtures arise when components in a population can be either weak or strong. A fundamental result for mixtures is that the mixture of components with decreasing failure rates have a decreasing failure rate. For convolutions, a basic result is that convolutions of lifetimes with increasing failure rates have increasing failure rates.

Other results on mixtures include the result that the asymptotic failure rate is the same as that of the failure rate of the strongest item, see Block et al. (1993), and also that the asymptotic direction of the failure rate corresponds to that of its strongest member, see Block and Joe (1997) and also Block et al. (2003). In particular, if the failure rate of the strongest component is eventually increasing, so is the failure rate of the mixture. Failure rates for mixtures with bathtub shape have been analyzed by Navarro and Hernandez (2004), Wondmagegnehu et al. (2005) and Navarro et al. (2009).

Received 23 May 2014; revision received 2 October 2014.

* Postal address: Department of Statistics, University of Pittsburgh, Pittsburgh, PA 15260, USA.

** Postal address: Department of Statistics, Haifa University, Haifa, 31905, Israel.

** Email address: hwb@pitt.edu

*** Postal address: Department of Statistics, University of Pittsburgh, Pittsburgh, PA 15260, USA. 
We begin a program for convolutions similar to the one for mixtures. The first step is to show that the failure rate for a convolution converges to the failure rate of the strongest component. This is covered in Section 2. Although the result is quite general, it does not cover the case when the failure rate of the strongest item is unbounded, such as in the case of a decreasing failure rate (DFR) gamma. This case will be considered in a separate paper; see Block et al. (2014).

In the case of mixtures, as mentioned above, we also know that the ultimate direction of its failure rate follows that of its strongest subpopulation. This does not hold for convolutions. We provide a counterexample in Section 3.

\section{The general case}

In this section we derive our general result. Consider the convolution of two life distributions having densities $g(t), h(t)$, survival functions $\bar{G}(t), \bar{H}(t)$, and failure rates $a(t), b(t)$. We assume that $\lim _{t \rightarrow \infty} a(t)=a$ and $\lim _{t \rightarrow \infty} b(t)=b$ with $0<a<b<\infty$. The density $f(t)$ and survival function $\bar{F}(t)$ of the convolution are respectively given by

$$
\begin{aligned}
& f(t)=\int_{0}^{\infty} g(t-y) \mathrm{d} H(y)=\int_{0}^{\infty} h(t-y) \mathrm{d} G(y), \\
& \bar{F}(t)=\int_{0}^{\infty} \bar{G}(t-y) \mathrm{d} H(y)=\int_{0}^{\infty} \bar{H}(t-y) \mathrm{d} G(y) .
\end{aligned}
$$

But since these are life distributions, $g(u)=h(u)=0$ and $\bar{G}(u)=\bar{H}(u)=1$ for $u<0$, these reduce to

$$
\begin{gathered}
f(t)=\int_{0}^{t} g(t-y) h(y) \mathrm{d} y=\int_{0}^{t} h(t-y) g(y) \mathrm{d} y, \\
\bar{F}(t)=\int_{0}^{t} \bar{G}(t-y) h(y) \mathrm{d} y+\bar{H}(t)=\int_{0}^{t} \bar{H}(t-y) g(y) \mathrm{d} y+\bar{G}(t) .
\end{gathered}
$$

Let $c(t)=f(t) / \bar{F}(t)$ be its failure rate. We want to show that $c(t)$ converges to the asymptotic limit of the strongest component, i.e. that $\lim _{t \rightarrow \infty} c(t)=a$. We first need a lemma.

Lemma 1. Let $r(t)$ be the failure rate of a life distribution with survival function $S(t)$. Assume that $\lim _{t \rightarrow \infty} r(t)=\alpha$ for $0<\alpha<\infty$. Then, for every $\delta, \eta$ satisfying $0<\delta<\alpha<\eta<\infty$, there exists constants $C_{1}, C_{2}$ such that

$$
C_{1} \mathrm{e}^{-\eta t} \leq S(t) \leq C_{2} \mathrm{e}^{-\delta t} \quad \text { for all } t \geq 0 .
$$

Proof. Fix $\delta, \eta$ as above and set $2 \varepsilon_{1}=\eta-\alpha, 2 \varepsilon_{2}=\alpha-\delta$. Since $r(t) \rightarrow \alpha$ as $t \rightarrow \infty$, there exists $t_{0}$ such that for all $t \geq t_{0}$,

$$
|r(t)-\alpha|<\varepsilon
$$

where $\varepsilon=\min \left\{\varepsilon_{1}, \varepsilon_{2}\right\}$. Hence, for $t \geq t_{0}$, we have

$$
\delta\left(t-t_{0}\right) \leq \int_{t_{0}}^{t} r(u) \mathrm{d} u \leq \eta\left(t-t_{0}\right) .
$$

Since we can write

$$
S(t)=\exp \left\{-\int_{0}^{t} r(u) \mathrm{d} u\right\}=S\left(t_{0}\right) \exp \left\{-\int_{t_{0}}^{t} r(u) \mathrm{d} u\right\},
$$


it follows that

$$
S\left(t_{0}\right) \mathrm{e}^{-\eta\left(t-t_{0}\right)} \leq S(t) \leq S\left(t_{0}\right) \mathrm{e}^{-\delta\left(t-t_{0}\right)} \quad \text { for all } t \geq t_{0} .
$$

Our result follows by setting

$$
C_{1}=\min \left\{c_{1}, S\left(t_{0}\right) \mathrm{e}^{\eta t_{0}}\right\}, \quad C_{2}=\max \left\{c_{2}, S\left(t_{0}\right) \mathrm{e}^{\delta t_{0}}\right\},
$$

where $c_{1}=\min _{\left\{0 \leq t \leq t_{0}\right\}}\left\{S(t) \mathrm{e}^{\eta t}\right\}$ and $c_{2}=\max _{\left\{0 \leq t \leq t_{0}\right\}}\left\{S(t) \mathrm{e}^{\delta t}\right\}$.

We use this lemma to conclude our main result.

Theorem 1. Consider the convolution of two life distributions $g(t), h(t)$ with survival functions $\bar{G}(t), \bar{H}(t)$ and failure rates $a(t), b(t)$ as described at the beginning of Section 1. Assume that $\lim _{t \rightarrow \infty} a(t)=a, \lim _{t \rightarrow \infty} b(t)=b$ with $0<a<b<\infty$ and that $a(t)$ is bounded. Then the failure rate of the convolution $c(t)$ converges to a as $t \rightarrow \infty$.

Proof. If for each $t>0$, we introduce the probability measure $M_{t}(\mathrm{~d} y)$,

$$
\begin{aligned}
M_{t}(\mathrm{~d} y) & =\frac{\bar{G}(t-y) h(y) \mathrm{d} y}{\bar{F}(t)} \\
& =\frac{\bar{G}(t-y) h(y) \mathrm{d} y}{\int_{0}^{\infty} \bar{G}(t-w) h(w) \mathrm{d} w} \\
& =\frac{\bar{G}(t-y) h(y) \mathrm{d} y}{\int_{0}^{\infty} \bar{H}(t-w) g(w) \mathrm{d} w} \\
& =\frac{\bar{G}(t-y) h(y) \mathrm{d} y}{\int_{0}^{t} \bar{G}(t-w) h(w) \mathrm{d} w+\bar{H}(t)} \\
& =\frac{\bar{G}(t-y) h(y) \mathrm{d} y}{\int_{0}^{t} \bar{H}(t-w) g(w) \mathrm{d} w+\bar{G}(t)},
\end{aligned}
$$

we can write

$$
c(t)=\int_{0}^{\infty} a(t-y) M_{t}(\mathrm{~d} y)
$$

and, hence,

$$
c(t)-a=\int_{0}^{\infty}[a(t-y)-a] M_{t}(\mathrm{~d} y) .
$$

In the above, we set $a(u)=0$ for all $u<0$ and use the fact that $g(t)=a(t) \bar{G}(t)$. Given $\varepsilon>0$, there exists $L>0$ such that $|a(u)-a|<\varepsilon$ for all $u \geq L$. We next choose positive numbers $\eta, \sigma$, and $\xi$ satisfying $a<\eta<\eta+\sigma<\xi<b$. Set $K(t)=((\eta+\sigma) / \xi) t$ and write

$$
c(t)-a=\int_{0}^{K(t)}[a(t-y)-a] M_{t}(\mathrm{~d} y)+\int_{K(t)}^{\infty}[a(t-y)-a] M_{t}(\mathrm{~d} y) .
$$

If $t>L /(1-(\eta+\sigma) / \xi)$, we can bound the first integral by $\varepsilon$ since then $L<t-K(t) \leq$ $t-y \leq t$ for all $0 \leq y \leq K(t)$.

We next use Lemma 1 to control the second integral. Since we are assuming that $a(t)$ is bounded, we can define the finite number $A=2 *\left(\sup _{\{0 \leq u<\infty\}}|a(u)|\right)$. Then

$$
\begin{aligned}
\left|\int_{K(t)}^{\infty}[a(t-y)-a] M_{t}(\mathrm{~d} y)\right| & \leq A \bar{M}_{t}[K(t)]=A \frac{\int_{K(t)}^{\infty} \bar{G}(t-y) h(y) \mathrm{d} y}{\int_{0}^{t} \bar{H}(t-w) g(w) \mathrm{d} w+\bar{G}(t)} \\
& \leq A \bar{H}[K(t)] / \bar{G}(t) .
\end{aligned}
$$


Here, as usual, $\bar{M}_{t}$ is the survival function of the probability distribution $M_{t}$. By Lemma 1 , since we have $a<\eta<\xi<b$ we know that there exists $C, D$ such that

$$
\bar{G}(t) \geq C \mathrm{e}^{-\eta t}, \quad \bar{H}[K(t)] \leq D \mathrm{e}^{-\xi K(t)} \quad \text { for all } t \geq 0 .
$$

Combining these two results and noting that $\eta t-\xi K(t)=\eta t-(\eta+\sigma) t=-\sigma t$, it follows that

$$
|c(t)-a| \leq \varepsilon+A\left(\frac{D}{C}\right) \mathrm{e}^{\eta t-\xi K(t)}=\varepsilon+\left(\frac{A D}{C}\right) \mathrm{e}^{-\sigma t} \quad \text { for all large } t,
$$

i.e. $\lim \sup _{t \rightarrow \infty}|c(t)-a| \leq \varepsilon$. This completes the proof.

Remark 1. Note that Theorem 1 does not cover the case when the strongest component has a failure rate which is unbounded, such as in the case of a DFR gamma density. This may just be a weakness of our method of proof. The case of a convolution of gamma distributions is considered in a separate paper (Block et al. (2014), where it is shown that the result of Theorem 1 remains true for all gamma distributions. The proof of this result, however, is completely different.

Corollary 1. Consider the convolution of $n$ independent lifetimes $X_{i}$ with failure rates $a_{i}(t)$, $i=1, \ldots, n$ and assume that $a_{i}(t) \rightarrow a_{i}$ as $t \rightarrow \infty$ with $0<a_{i}<\infty$. If $a_{1}<$ $\min \left\{a_{2}, \ldots, a_{n}\right\}$ and $a_{1}(t)$ is bounded, then the failure rate of the convolution converges to $a_{1}$ as $t \rightarrow \infty$.

Proof. The proof follows easily. By Theorem 1, the failure rate of $X_{1}+X_{2}$ converges to $a_{1}$. Next, we write $X_{1}+X_{2}+X_{3}$ as $\left(X_{1}+X_{2}\right)+X_{3}$. It follows from (1) that the failure rate of $X_{1}+X_{2}$ is bounded; hence, the failure rate of $X_{1}+X_{2}+X_{3}$ converges to $a_{1}$. Continue by induction.

\section{A counterexample}

In the preceeding section we showed that the failure rate of a convolution converges to the limiting failure rate of its strongest component, just as in the mixture case. For the mixture case, however, it was also shown Block et al. (2003) that the ultimate direction of the asymptotic

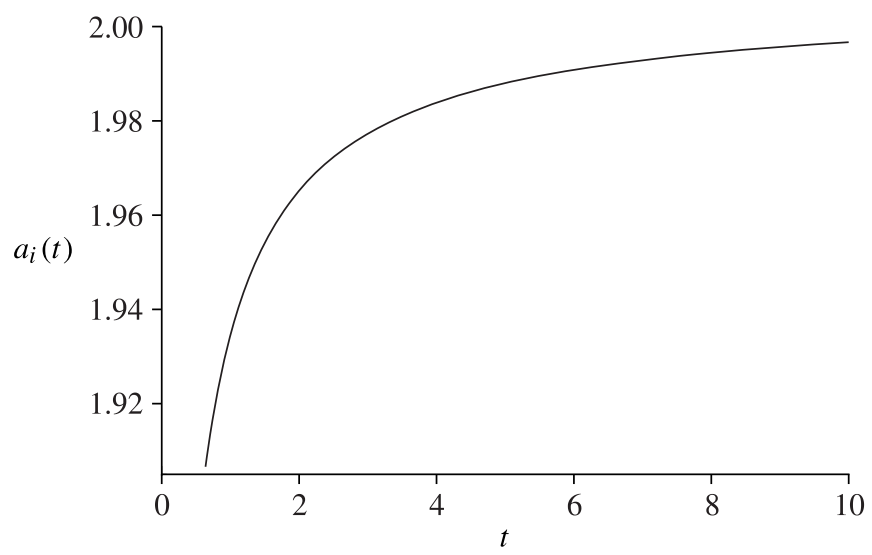

FIGURE 1: Counterexample. 
failure rate followed that of its strongest subpopulation. The example below shows that this is not generally the case for convolutions.

A clue as to why this is not true is to consider the convolution of two DFR gamma densities with shape parameters $0<\alpha_{1}<\alpha_{2}<1$ but with the same scale parameter $\lambda$. The convolution is thus a gamma with shape parameter $\alpha_{1}+\alpha_{2}$ and scale parameter $\lambda$. If $\alpha_{1}+\alpha_{2}>1$ then the failure rate of the convolution is IFR and, hence, increases to $\lambda$, even though the failure rate of both its component are decreasing. Although this example does not provide a direct counterexample since the limiting failure rates of both components are the same $\lambda$, a slight variation does.

Example 1. Consider the convolution of two DFR gamma densities having shape parameters $\alpha_{1}=0.4, \alpha_{2}=0.7$, and scale parameters $\lambda_{1}=2, \lambda_{2}=2.01$. The MATHEMATICA ${ }^{\circledR}$ plot in Figure 1 shows that the failure rate of the convolution increases to 2 .

\section{Acknowledgement}

We would like to thank Sheldon Ross for suggesting the problem.

\section{References}

Block, H. AND Joe, H. (1997). Tail behavior of the failure rate functions of mixtures. Lifetime Data Anal. 3, $269-288$.

Block, H. W., Langberg, N. A. And Savits, T. H. (2014). The limiting failure rate for a convolution of gamma distributions. Prob. Statist. Lett. 94, 176-180

Block, H. W., LI, Y. AND SAVITS, T. H. (2003). Initial and final behaviour of failure rate functions for mixtures and systems. J. Appl. Prob. 40, 721-740.

Block, H. W., Mi, J. And SAVITs, T. H. (1993). Burn-in and mixed populations. J. Appl. Prob. 30, 692-702.

LaI, C.-D. AND XIE, M. (2006). Stochastic Ageing and Dependence for Reliability. Springer, New York.

Marshall, A. W. and Olkin, I. (2007). Life Distributions. Springer, New York.

Navarro, J. and Hernandez, P. J. (2004). How to obtain bathtub-shaped failure rate models from normal mixtures. Prob. Eng. Inf. Sci. 18, 511-531.

Navarro, J., Guillamon, A. and Ruiz, M. C. (2009). Generalized mixtures in reliability modelling: applications to the construction of bahtub shaped hazard models and the study of systems. Appl. Stoch. Models Business Industry. 25, 323-337.

Wondmagegnehu, E. T., Navarro, J. and Hernandez, P. J. (2005). Bathtub shaped failure rates from mixtures: a practical point of view. IEEE Trans. Reliab. 54, 270-275. 